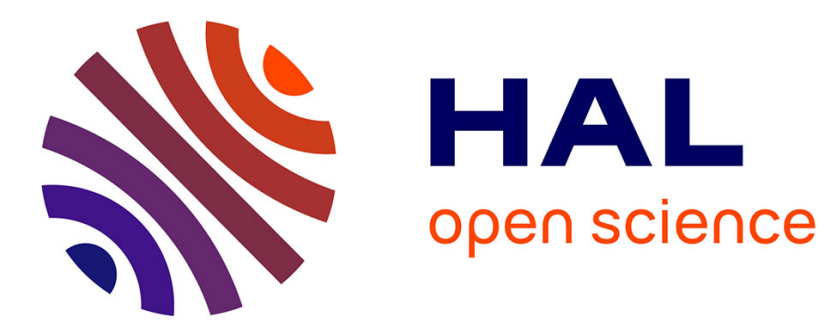

\title{
Three-dimensional instabilities and negative eddy viscosity in thin-layer flows
}

Alexandros Alexakis

\section{To cite this version:}

Alexandros Alexakis. Three-dimensional instabilities and negative eddy viscosity in thin-layer flows. Physical Review Fluids, 2018, 3 (11), pp.114601. 10.1103/PhysRevFluids.3.114601 . hal-02017806

\section{HAL Id: hal-02017806 \\ https://hal.sorbonne-universite.fr/hal-02017806}

Submitted on 13 Feb 2019

HAL is a multi-disciplinary open access archive for the deposit and dissemination of scientific research documents, whether they are published or not. The documents may come from teaching and research institutions in France or abroad, or from public or private research centers.
L'archive ouverte pluridisciplinaire HAL, est destinée au dépôt et à la diffusion de documents scientifiques de niveau recherche, publiés ou non, émanant des établissements d'enseignement et de recherche français ou étrangers, des laboratoires publics ou privés. 


\title{
3D instabilities and negative eddy viscosity in thin-layer flows
}

\author{
Alexandros AlEXAKIS* \\ Laboratoire de Physique Statistique, École Normale Supérieure, \\ PSL Research University; Université Paris Diderot Sorbonne Paris-Cité; Sorbonne \\ Universités UPMC Univ Paris 06; CNRS; 24 rue Lhomond, 75005 Paris, France
}

(Dated: October 11, 2018)

The stability of flows in layers of finite thickness $H$ is examined against small scale three dimensional (3D) perturbations and large scale two-dimensional (2D) perturbations. The former provide an indication of a forward transfer of energy while the latter indicate an inverse transfer and the possibility of an inverse cascade. The analysis is performed using a Floquet-Bloch code that allows to examine the stability of modes with arbitrary large scale separation. For thin layers the 3D perturbations become unstable when the layer thickness $H$ becomes larger than $H>c_{1}\left(\nu \ell_{U} / U\right)^{1 / 2}=c_{1} \ell_{U} R e^{-1 / 2}$, where $U$ is the rms velocity of the flown, $\ell_{U}$ is the correlation length scale of the flow, $\nu$ the viscosity and $R e=\ell_{U} U / \nu$ is the Reynolds number. At the same time large scale $2 \mathrm{D}$ perturbations also become unstable by an eddy viscosity mechanism when $R e>c_{2}$, where $c_{1}, c_{2}$ are order one non-dimensional numbers. These relations define different regions in parameter space where $2 \mathrm{D}$ and $3 \mathrm{D}$ instabilities can (co-)exist and this allows to construct a stability

diagram. Implications of these results for fully turbulent flows that display a change of direction of cascade as $H$ is varied are discussed.

PACS numbers: 47.20.-k,47.11.St,47.11.Kb,47.15.Fe, 


\section{INTRODUCTION}

In many systems in nature there is a transfer of energy to both large and small scales [1-10]. This transfer at large Reynolds numbers leads to a split cascade of energy such that part of the energy cascades to the small scales and gets dissipated while at the same time part of the energy cascades to the large scales typically forming large scale coherent structures [11-18]. For a recent review and classification of such split cascades see [19]. A typical example of such a behavior is observed in the Earth's atmosphere where, due to the combined effects of confinement, rotation and stratification, there is this split cascade leading to both small scale turbulence and fast mixing as well as large-scale structures like zonal flows and hurricanes. This split cascade has been quantified by in situ aircraft measurements in the hurricane boundary layer [20]. In the presence of a split cascade the amplitude of the inverse cascade depends on a control parameter, whose variation, makes the system transition from a state exclusively cascading energy to the small scales to a state that the energy cascade is split or strictly inverse. The simplest example perhaps is turbulence in layers of finite thickness $H$ such that at scales $L$ much larger than the thickness, $L \gg H$, the flow looks like two dimensional (2D) while at scales $\ell$ much smaller than the thickness, $H \gg \ell$, the flow behaves like a three dimensional (3D) flow. Depending on the relative value of $H$ with respect to the forcing scale $\ell_{U}$ the system can behave like $3 \mathrm{D}$ cascading energy forward or like 2D cascading energy inversely. The amplitude of the inverse cascade appears to decrease as $H / \ell_{U}$ is increased until a critical value is met $H_{c} / \ell_{U}$ such that the inverse cascade becomes exactly zero.

The mechanisms however under which such a coexistence of counter directed cascades exist are not yet understood. In this work we try to unravel some of the mechanisms involved by examining the stability properties of simple laminar flows to both large and small scale perturbations. Although such an approach can not be used to study the full nonlinear dynamics of a split cascade it provides a much simpler setup that can shed light on the mechanisms involved in the transfer of energy to small or larger scales. On the one hand it is known that 3D instabilities of laminar flows generate in general smaller scales transferring energy to them. On the other hand 2D instabilities can couple the forced modes to large scale 2D modes transferring energy to larger scales. This non-local interaction of scales can be quantified with the use of an eddy-viscosity that can change sign depending on the flow parameters. A possible way to understand the mechanisms involved in the transition from a forward to an inverse cascade is to examine when 3D instabilities dominate and when there is a change of sign for the eddy viscosity of the flow.

The notion of eddy viscosity has been introduced in turbulence very early by Taylor [21]. First attempts for its calculation were made by Kraichnan [22] in an attempt to quantify the loss or gain of energy to the small scales. In this framework the evolution of a weak large scale flows $\mathbf{v}$ due to small scale turbulent fluctuations $\mathbf{U}$ can be described as

$$
\partial_{t} v^{i}=-\sum_{j, k, m}\left(\nu \delta^{i, j} \delta^{l, m}+\nu_{e d d y}^{i, j, l, m}\right) \nabla^{l} \nabla^{m} v^{j}
$$

where $\nu$ is the regular viscosity and $\delta^{i, j}$ stands for the Kronecker delta. The tensor $\nu_{e d d y}^{i, j, l, m}$ is the eddy viscosity that models the effect of the small scale fluctuations $\mathbf{U}$ on $\mathbf{v}$. Since the work of Kraichnan there have been many attempts to calculate an eddy viscosity for turbulent flows [23-29]. However the cascade processes of turbulent flows that excites a continuous spectrum of scales prevents from having a closed expression for the eddy viscosity and even the notion of eddy viscosity for turbulent flows can be questioned. One case where the eddy viscosity tensor $\nu_{e d d y}^{i, j, l, m}$ can be rigorously defined is in the presence of large scale separation between the forced small scale field $\mathbf{U}$ that evolves at scales $\ell_{U}$ and the flow velocity $\mathbf{u}$ that evolves at scales $L \gg \ell_{U}$. It can then be calculated in the low Reynolds number limit using homogenization theory [30,31]. Here the Reynolds number is defined as $R e \equiv U \ell_{U} / \nu$ where $U$ is the rms of the small scale velocity field and $\ell_{U}$ is the typical length-scale of the flow. In the small Reynolds number limit and for isotropic flows it takes the form

$$
\nu_{e d d y}^{i, j, l, m}=\frac{c_{1}}{\nu} U^{2} \ell_{U}^{2}\left[\delta^{i, j} \delta^{l, m}+\mathcal{O}(R e)\right] \quad \text { for } \quad R e \ll 1,
$$

where $c_{1}$ is a non-dimensional number that depends on the detailed structure of the small scale field $\mathbf{U}$. For large $R e$ a viscosity independent value of $\nu_{e d d y}$ is expected to be reached

$$
\nu_{e d d y}^{i, j, l, m}=c_{2} U \ell_{U}\left[\delta^{i, j} \delta^{l, m}+\mathcal{O}\left(\frac{1}{R e}\right)\right] \quad \text { for } \quad R e \gg 1 .
$$

If $-\nu_{e d d y}^{i, j, l, m}$ has positive eigenvalues that are larger than the viscosity then the system can develop large scale instabilities [32].

An other possibility for the effect of small scales on the large is the anisotropic kinetic alpha effect [33]. This effect is similar to the alpha effect in dynamo theory, and leads to a faster growth-rate compared to a negative eddy viscosity. 
However, unlike the dynamo problem that requires only the presence of helicity, in hydrodynamics in order for the anisotropic kinetic alpha effect to be present the flow at small scales needs to satisfy much stricter conditions that are not met in the examined flows in this work. Fot this reason this work is only going to consider the effect of negative eddy viscosity as a large scale instability mechanism.

In practice it is not always feasible to calculate $\nu_{e d d y}$ analytically, and calculations are limited to either very simple flows [34-37] or in the low Re limit where the eddy-viscosity is sub-dominant to the regular viscosity. In this work a different path is followed by calculating the growth-rate of small and large scale instabilities using Floquet-Bloch theory from which the value of the eddy viscosity can be extracted. By doing so we can determine for the examined flows when the regions in the parameter space that the eddy viscosity is negative and large scale instabilities are present, and where the flow is dominated by three dimensional small scale instabilities instead.

\section{FORMULATION}

We begin by considering the flow of a unit density fluid in a triple periodic box of size $(2 \pi L, 2 \pi L, 2 \pi H)$ with $L \gg H$ so that the layer has a small height compared to its length in the other directions. The triple periodic box although unphysical it simplifies significantly the following calculations. No significant differences are expected from a flow in layers with free slip (stress-free) boundary conditions. For no-slip boundary conditions however the drag force from the boundary can have a significant effect on the dynamics. The flow satisfies the Navier-Stokes equation that is given by:

$$
\partial_{t} \mathbf{u}+\mathbf{u} \cdot \nabla \mathbf{u}=-\nabla P+\nu \mathbf{F}+\nu \Delta \mathbf{u}
$$

Here, $\mathbf{u}$ is the fluid velocity, $\nu$ is the viscosity and $\mathbf{F}$ is an external body force that maintains the flow. We assume the $\mathbf{F}$ is such that it supports a laminar time independent solution for the fluid $\mathbf{U}$.

$$
\mathbf{F}=-\nu \Delta \mathbf{U}+\mathbf{U} \cdot \nabla \mathbf{U}-\nabla P
$$

For simplicity a simple family of flows is going to be considered here, given by

$$
\mathbf{U}=U \cos \left(\frac{r \pi}{2}\right)\left[\begin{array}{c}
\sin \left(k_{U} y\right) \\
\sin \left(k_{U} x\right) \\
0
\end{array}\right]+\sqrt{2} U \sin \left(\frac{r \pi}{2}\right)\left[\begin{array}{c}
\sin \left(k_{U} y\right) \\
\sin \left(k_{U} x\right) \\
0
\end{array}\right] \sin \left(k_{U} z\right)
$$

where $k_{U}=1 / \ell_{U}$ is the wavenumber of the flow and $U=\|\mathbf{U}\|$ is the $L_{2}$ norm of the flow. Note that $U$ is independent of the parameter $r$. For the remainder of this investigation we will set $k_{U}=1$ and $U=1$. With this normalization the Reynolds number of the flow is always given by

$$
R e=\frac{U \ell_{U}}{\nu}=\frac{1}{\nu}
$$

For $r=0$, the flow is a two dimensional (2D) cell flow and is a solution of the Euler equations. For $r \neq 0$, $\mathbf{U}$ is three dimensional (3D) as it varies in all three dimensions. For $r=1$ the flow reduces to a Taylor-Green flow [38] a prototypical flow that was used to study the small scale generation. Note that if $r$ is not zero $\mathbf{U}$ is not a solution of the Euler equations and $\mathbf{F}$ has to have a more complex behavior than $\mathbf{U}$ to sustain it against viscosity and the non-linearities. Furthermore we note that for $r=0, H$ can take any value, while for $r \neq 0, H$ is restricted to be an integer multiple of the flow period $H=n \ell_{U}$ with $n$ an integer.

The main objective of this work is to examine the stability of this flow and examine the evolution of an infinitesimal perturbation $\mathbf{v}$ to the laminar flow so that $\mathbf{u}=\mathbf{U}+\mathbf{v}$. The linear evolution equation for $\mathbf{v}$ reads

$$
\partial_{t} \mathbf{v}+\mathbf{U} \cdot \nabla \mathbf{v}=-\mathbf{v} \cdot \nabla \mathbf{U}-\nabla P+\nu \nabla^{2} \mathbf{v}
$$

The linearity and homogeneity in time of the problem implies that asymptotically at large times $\mathbf{v}$ will have an exponential behavior with time and the goal is to determine the growth rate $\gamma$ of the perturbation in terms of the parameters of the system. Because $\mathbf{U}$ is periodic in space and we are interested in the behavior of scales much larger than the scale of $\mathbf{U}$ it is convenient to use Floquet (or Bloch) theory. Floquet theory states that if $\mathbf{U}$ is periodic the perturbation field $\mathbf{v}$ can be decomposed as

$$
\mathbf{v}(\mathbf{x}, t)=e^{i \mathbf{q} \cdot \mathbf{x}} \tilde{\mathbf{v}}(\mathbf{x}, t)+c . c .
$$


where $\tilde{\mathbf{v}}(\mathbf{x}, t)$ is a complex vector field that has the same spatial periodicity as the velocity field $\mathbf{U}$, and $\mathbf{q}$ is an arbitrary wave number. The linear evolution equation for the field $\tilde{\mathbf{v}}(\mathbf{x}, t)$ then becomes

$$
\partial_{t} \tilde{\mathbf{v}}+\mathbf{U} \cdot \nabla \tilde{\mathbf{v}}+i \mathbf{U} \cdot \mathbf{q} \tilde{\mathbf{v}}=-\tilde{\mathbf{v}} \cdot \nabla \mathbf{U}-\nabla \tilde{P}+\nu(\nabla+i \mathbf{q})^{2} \tilde{\mathbf{v}}
$$

where the pressure $\tilde{P}$ is such so that it guarantees the incompressibility condition $\nabla \tilde{\mathbf{v}}+i \mathbf{q} \cdot \tilde{\mathbf{v}}=0$. The advantage of studying eq. (9) numerically as opposed to eq. (7) is that one can consider arbitrary large scale separations (determined by the vector parameter $\mathbf{q}$ ) with no additional computational cost. Furthermore, the Floquet formulation gives a clear distinction between small scale and large scale instabilities. For $q=|\mathbf{q}|\left\langle k_{U}\right.$, the volume average $\langle\tilde{\mathbf{v}}\rangle$ over the spatial period $\left(2 \pi \ell_{U}\right)^{3}$ gives the amplitude of $\mathbf{v}$ at large scales $L \propto 2 \pi / q\left(\right.$ ie $\langle\mathbf{v}\rangle=\langle\tilde{\mathbf{v}}\rangle e^{i \mathbf{q} \cdot \mathbf{x}}+$ c.c. $)$. Fields with $q=0$ and/or $\langle\tilde{\mathbf{v}}\rangle=0$ correspond to purely small scale fields, and if these modes are unstable it amounts to a pure small scale instability $i e$ : an instability that involves only wavenumbers that are integer multiples of the basic period $k_{U}$.

Eq. (9) is solved numerically using a pseudospectral code. Details of the code can be found in [32, 39]. The resolutions examined varied from $32^{3}$ grid points for most of the results here although resolutions of $64^{3}$ grid points and $128^{3}$ grid points were also used for large $R e$ and to test convergence.

\section{TWO DIMENSIONAL FLOWS $r=0$}

We begin by examining the case for which $r=0$ so that the laminar flow is two-dimensional. In this case the laminar flow can be written in terms of a stream function $\Psi=\cos \left(k_{U} x\right)-\cos \left(k_{U} y\right)$ as $U=\left[\partial_{y} \Psi,-\partial_{x} \Psi, 0\right]$, where the stream function is connected to the vertical vorticity $W$ as $W=-\nabla^{2} \Psi=k_{U}^{2} \Psi$. The last equality holds because $\mathbf{U}$ contains modes of single wavenumber $k_{U}$. The stability of this $2 \mathrm{D}$ flow will be examine in the next subsections, first against 2D perturbations and second against 3D perturbations. The stability for 2D perturbations has been examined before in [40] however in the presence of large scale linear friction that does not allow to directly compare with these results.

\section{A. Two dimensional perturbations}

For velocity perturbations that are also $2 \mathrm{D}$ (ie $\partial_{z} \mathbf{v}=\mathbf{0}$ ) the vertical component of the perturbation velocity decouples and follows a passive advection diffusion equation

$$
\partial_{t} v_{z}+\mathbf{U} \cdot \nabla v_{z}=\nu \nabla^{2} v_{z}
$$

As a consequence its $L_{2}$ norm $\left\|v_{z}\right\|^{2}$ follows

$$
\partial_{t}\left\|v_{z}\right\|^{2}=-\nu\left\|\nabla v_{z}\right\|^{2} \leq-\nu\left(\frac{2 \pi}{L}\right)^{2}\left\|v_{z}\right\|^{2}
$$

and therefore the norm $\left\|v_{z}\right\|^{2}$ decays monotonically with time. The remaining two components $\mathbf{v}_{\mathbf{2 D}}=\left[v_{x}, v_{y}, 0\right]$ can be written in terms of a stream function $\psi$ as $v_{x}=\partial_{y} \psi, v_{y}=-\partial_{x} \psi$. The evolution of these components can be written in terms of their vorticity $w=-\nabla^{2} \psi$ as

$$
\partial_{t} w+\mathbf{U} \cdot \nabla w=-\mathbf{v}_{\mathbf{2} \mathbf{D}} \cdot \nabla W-\nu \nabla^{2} w
$$

Multiplying eq. (11) by $w$ and space averaging we obtain after some manipulation (and using the laminar flow property $\left.W=-\nabla^{2} \Psi=k_{U}^{2} \Psi\right)$ the enstrophy evolution equation

$$
\partial_{t}\|w\|^{2}=k_{U}^{2}\langle w \mathbf{U} \cdot \nabla \psi\rangle-\nu\|\nabla w\|^{2}
$$

Multiplying eq. (11) by $\psi$ and space averaging we obtain after similar manipulations the 2D energy evolution equation

$$
\partial_{t}\left\|\mathbf{v}_{2 D}\right\|^{2}=-\langle\psi \mathbf{U} \cdot \nabla w\rangle-\nu\|w\|^{2} .
$$

Multiplying the energy evolution equation by $k_{U}^{2}$ and subtracting from the enstrophy equation we obtain

$$
\partial_{t}\left(\|w\|^{2}-k_{U}^{2}\left\|\mathbf{v}_{2 D}\right\|^{2}\right)=-\nu\left(\|\nabla w\|^{2}-k_{U}^{2}\|w\|^{2}\right)
$$

In general, the terms in parenthesis on the left and right hand side of the equation above 14 can have either sign and the equation above can not exclude the growth of $w$. If however $k_{U}=2 \pi / L$ so that the domain size $L$ coincides 
with the velocity scale $2 \pi k_{U}^{-1}$ (ie no scales larger than the forcing scale are allowed) both $\left(\|w\|^{2}-k_{U}^{2}\left\|\mathbf{v}_{2 D}\right\|^{2}\right.$ ) and $\left(\|\nabla w\|^{2}-k_{U}^{2}\|w\|^{2}\right)$ are non-negative (by Poincaré inequality). The right hand side of eq. (14) is then negative and the positive quantity $\left(\|w\|^{2}-k_{U}^{2}\left\|\mathbf{v}_{2 D}\right\|^{2}\right)$ has to decrease monotonically. This implies that single scale 2D flows such that $\nabla^{2} \Psi=-k_{U}^{2} \Psi$ where $k_{U}$ is the largest scale of the domain are linearly stable to all $2 \mathrm{D}$ perturbations. In fact eq. 12 and 13 also hold for the nonlinear evolution of $\mathbf{w}$ and $\mathbf{v}_{2 D}$ and the flow is thus non-linearly stable to all 2D perturbations as shown for the first time in [41]. In terms of the Floquet decomposition given in eq. (8) this conclusion translates to: the flow is stable for $q=0$ ie there are no small scale instabilities, and any unstable mode has to excite simultaneously scales both smaller and larger than the forcing.

Given this restriction we investigate numerically eq. (9) using a pseudo spectral code (described in [32, 39]) and calculate the growth-rate $\gamma$ of the unstable modes as a function of $q$ and the viscosity $\nu$. The procedure to calculate this growth-rate is as follows. We chose the wave-vector $\mathbf{q}=\left[q_{x}, 0,0\right]$. In this case since we are interested in $2 \mathrm{D}$ perturbations $\mathbf{q}$ is restricted in the $x, y$ plane. For simplicity, results are presented only for $\mathbf{q}=\left[q_{x}, 0,0\right]$ that was found (but not proven) to be the most unstable from the general cases examined $\mathbf{q}=\left[q_{x}, q_{y}, 0\right]$. For this chosen wave-vector $\mathbf{q}=\left[q_{x}, 0,0\right]$ the complex velocity field $\tilde{\mathbf{v}}$ is initialized using random initial conditions. Then the complex field $\tilde{\mathbf{v}}$ is evolved based on eq. 9 until a clear exponential growth of the energy $E=\left\langle|\tilde{\mathbf{v}}|^{2}\right\rangle \propto e^{2 \gamma t}$ is observed. The growth-rate $\gamma$ is then the calculated by fitting. This procedure is then repeated for different values of $q$ and different values of viscosity. This allows to calculate the growth-rate of the most unstable mode as a function of $q$ and $\nu$. It is noted that this procedure reveals only the fastest growing unstable mode and not all unstable modes in the system. It is worth keeping this in mind in the following sections where general 3D perturbations will be considered.
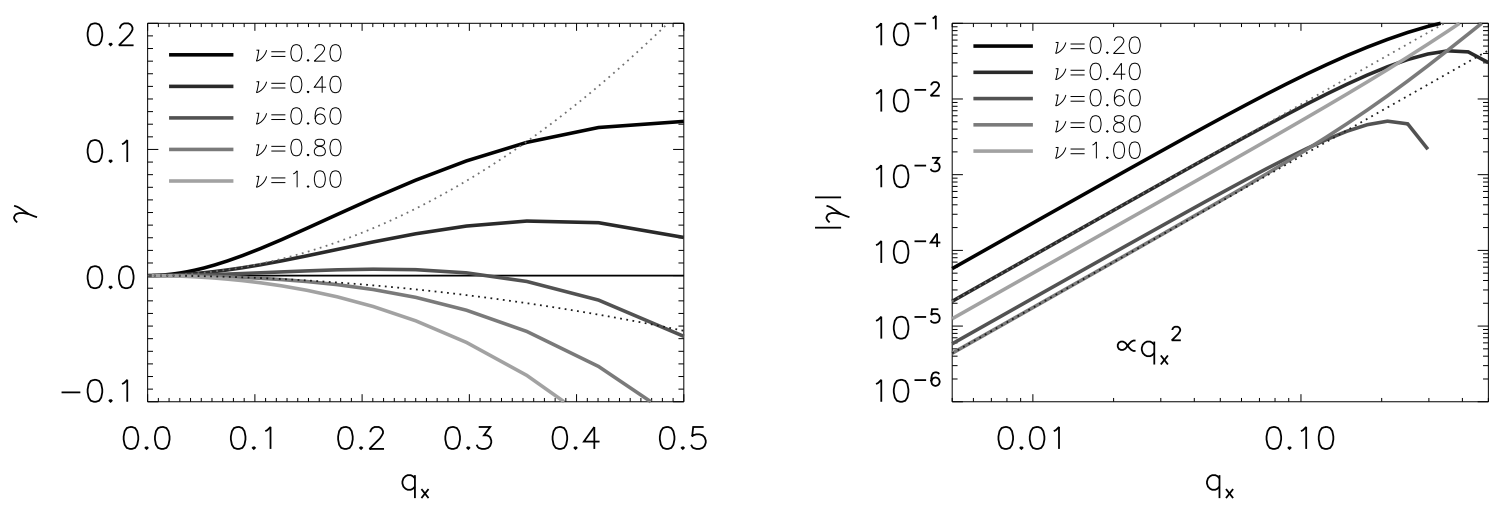

Figure 1. The growth rate $\gamma$ as a function of the wavenumber $q$ for five different values of the the viscosity $\nu$. The dotted lines show fits to a quadratic power-law $\gamma=a q^{2}$. Left panel: linear scale. Right panel: The absolute value of $\gamma$ in log log scale.

The resulting growth rate $\gamma$ as a function of $q$ for different values of the viscosity is shown in the left panel of figure 1 in linear scale. The right panel shows the absolute value of the same data in logarithmic scale. As discussed in the beginning of this section for $q=0$ there can be no instabilities and the growth rate becomes zero as $q \rightarrow 0$. The growth rate $\gamma$ is approaching zero as $q \rightarrow 0$ following a quadratic power-law. This is demonstrated more clearly in logarithmic scale shown in the right panel of figure 1 where it is fitted as

$$
\gamma=a q^{2}
$$

shown by the dotted lines. The proportionality coefficient $a$ is negative for large values of the viscosity, while bellow a critical value it becomes positive. For larger $q$ higher even powers (due to symmetry) of $q$ are needed to describe $\gamma=a q^{2}+\beta q^{4}+\ldots$ and as $\nu$ is decreased the maximum growth-rate moves to larger $q$. At $\nu$ sufficiently small the most unstable mode is found at $q=1 / 2$, indicating that the wavenumbers close to the forcing one are the most unstable. Nonetheless, the onset of the large scale instability is determined by the sign of the pre-factor $a$ of the quadratic power-law in eq. 15. This particular scaling of the growth-rate $\gamma$ with the wavenumber $q$ given in eq. 15 implies that the instability is of the form of an eddy-viscosity. The eddy viscosity is a tensor and the proportionality coefficient $a$ in eq. (15) indicates the maximum eigenvalue of $-\left(\nu \delta_{i, j}+\nu_{e d d y}^{i, j, x, x}\right)$. For simplicity $a+\nu$ will be denoted as $-\nu_{e d d y}$ and referred as eddy viscosity although it must not be forgotten that the eddy viscosity is truly a tensor. The relation of $\nu_{e d d y}$ with $a$ is

$$
a=-\left(\nu+\nu_{e d d y}\right)
$$


With this notation a large scale instability is implied if $\nu_{e d d y}<-\nu$.

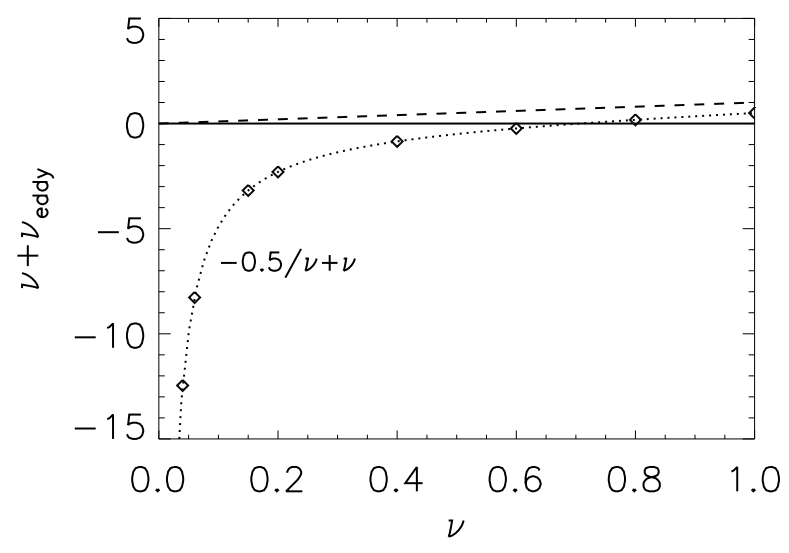

Figure 2. The value of $\nu_{e d d y}+\nu$ as a function of $\nu$. When this quantity takes negative values there is a large scale instability. The dashed line indicates where $\nu_{e d d y}=0$. The doted line shows the fitting to the asymptotic result $\nu_{e d d y}=-0.5 / \nu$. Large scales become unstable when $\nu<\nu^{*} \simeq 0.7$.

The eddy viscosity depends on the small-scale flow examined and the value of viscosity. Figure 2 shows the value of $\nu+\nu_{e d d y}$ as a function of $\nu$ for the examined flow. The dotted lines indicates the prediction (2) for $c_{1}=0.5$. The fit to the power-law is surprisingly good even up to $\nu \simeq 0.05$ which is far from the asymptotic regime $R e \ll 1$. Negative values of $\nu+\nu_{e d d y}$ imply that the flow amplifies large scale flows. For large values of $\nu$ the value of the eddy-viscosity is such that $\nu+\nu_{e d d y}$ is positive although still smaller than $\nu$. So although $\nu_{e d d y}$ is negative and the flow reduces the decay rate caused by regular viscosity it is not sufficiently strong to drive any large scale scale instabilities. Bellow some critical value of the viscosity $\nu=\nu^{*}$ the value of $\nu+\nu_{\text {eddy }}$ becomes negative. For values of $\nu$ smaller than $\nu^{*}$ the large scales of the flow are unstable. The critical value of the viscosity $\nu^{*}$ can be estimated to be

$$
\nu^{*} \simeq 0.7 U \ell_{U}
$$

where we have kept $U=1$ and $\ell_{U}=1$ in the expression to recover the dimensions.

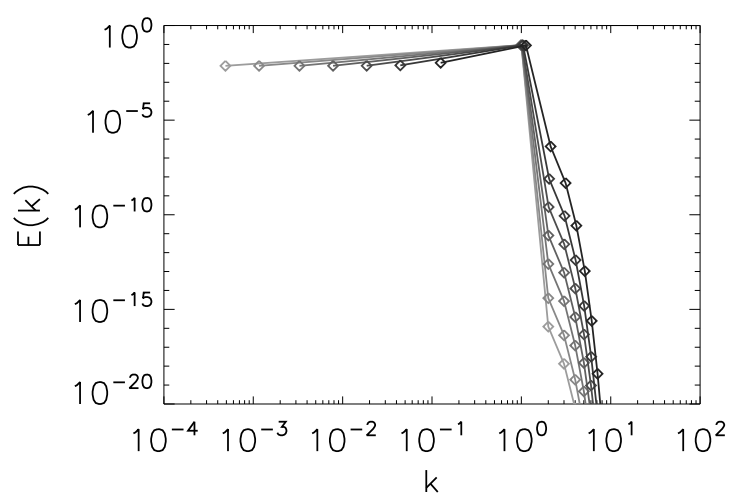

Figure 3. Energy spectrum of the unstable modes $e^{i \mathbf{q} \cdot \mathbf{x}} \tilde{\mathbf{v}}(\mathbf{x}, t)$ for different values of $q$ and $\nu=0.2$, and $k_{U}=1$ is the wavenumber of the laminar flow. The value of $q$ is indicated by the furthest point to the left.

Before concluding this section it is worth examining the spectral shape of the unstable modes. Figure 3 shows the energy spectra of the unstable modes as a function of the wavenumber for different values of $q$. The spectrum is composed by the large scale component located at the wave number $q$ plus the part localized at small scales $k \geq k_{U}=1$. The amplitude of the energy at large scales is independent on the value of $q$. This is seen in the figure 4 where the ratio of the energy contained in the largest scale $E_{0}=\frac{1}{2}|\langle\tilde{\mathbf{v}}\rangle|^{2}$ to the total energy $E=\frac{1}{2}\left\langle|\tilde{\mathbf{v}}|^{2}\right\rangle$ is shown. 

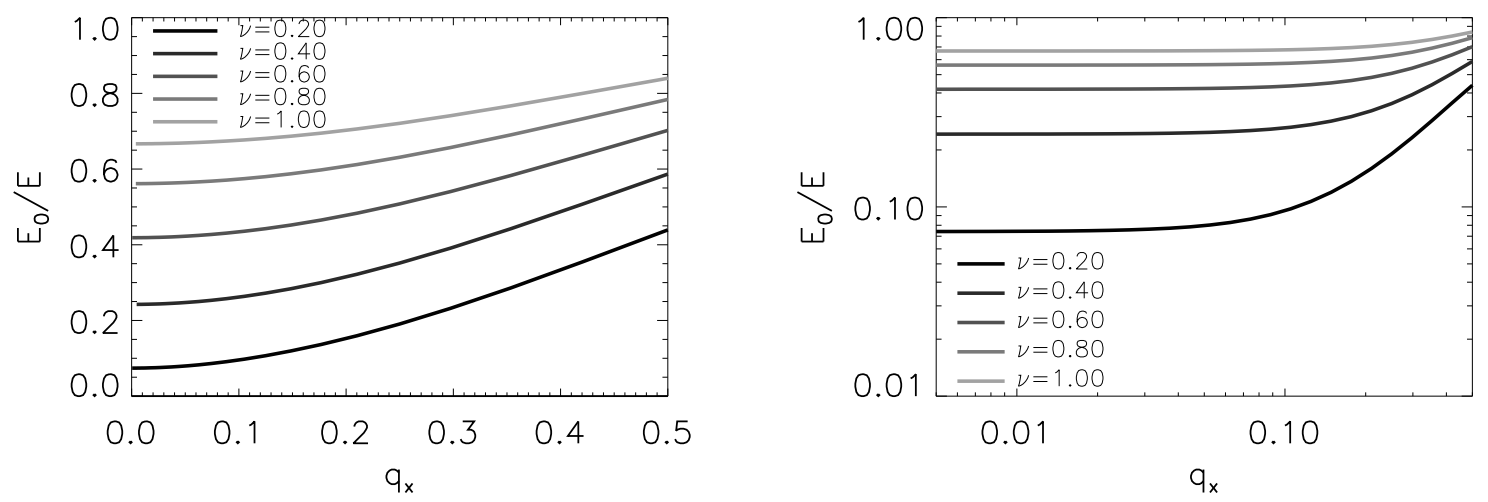

Figure 4. The ratio of the energy in the large scales mode $E_{0}=\frac{1}{2}|\langle\tilde{\mathbf{v}}\rangle|^{2}$ to the total energy $E=\frac{1}{2}\left\langle|\tilde{\mathbf{v}}|^{2}\right\rangle$ as a function of $q$ for different values of $\nu$. Left: on a linear scale, right in log-log scale.

Note that $E_{0} / E$ is shown also for modes with negative growth rate. The amplitude of this ratio is initially decreased as $q$ is decreased but asymptotes to a $q$ independent value for sufficiently small values of $q$. The fact that the $q=1 / 2$ modes have a larger value of $E_{0} / E$ than small $q$ also indicates that the $q=1 / 2$ are more efficient at extracting energy from the forcing scale. This asymptotic value at small $q$ decreases as $\nu$ decreases, which can be shown in the asymptotic expansion in [30].

\section{B. Three dimensional perturbations}

In the previous section we examined the stability of the $2 \mathrm{D}$ flow against large scale $2 \mathrm{D}$ perturbations. In this section we investigate the stability of the flow against 3D perturbations. This question is important because it determines when $3 \mathrm{D}$ variations enter the system that can transfer energy to the small scales.

For $r=0$ the flow $\mathbf{U}$ has no dependence on the $z$ direction. We can therefore consider a layer with arbitrary thickness compared to the flow length-scale $k_{U}^{-1}$. The homogeneity in the $z$-direction also implies that each $q_{z}$ mode can be considered independently for the linear problem with the perturbation field $\tilde{\mathbf{v}}$ being a function of $x, y, t$ only and not of $z$. This section is restricted to $3 \mathrm{D}$ perturbations with wavenumbers $\mathbf{q}=\left[0,0, q_{z}\right]$. For this choice the field $\tilde{\mathbf{v}}$ satisfies the incompressibility condition

$$
\partial_{x} \tilde{v}_{x}+\partial_{y} \tilde{v}_{y}+i q_{z} \tilde{v}_{z}=0
$$

Note that unlike the previous case of $2 \mathrm{D}$ perturbations (with $q_{z}=0$ ) the incompressibility condition implies that the $z$-component does not act independently.

Figure 5 displays the growth rate $\gamma$ as a function of $q_{z}$ for different values of the viscosity $\nu$. Bellow a critical value of $\nu<\nu_{0} \simeq 0.1$ modes with positive growth rates appear with $q_{z} \propto k_{U}=1$. As $\nu$ is decreased further the range of unstable wave-numbers extends to larger and large values of $q_{z}$. For $\nu \ll \nu_{0}$ the unstable wavenumbers are found in the range $q_{z}<q_{z}^{*}$, here $q_{z}^{*}$ is the largest unstable wavenumber. This critical wavenumber $q_{z}^{*}$ can be estimated from the graph in the left panel of figure 5 as the wavenumber for which the growth rate intersects the zero growth rate $\gamma=0$ line. It depends on $\nu$ and it is plotted in the right panel of figure 5 in a logarithmic scale. It is shown to follow the scaling $q_{z}^{*} \propto \nu^{-1 / 2}$ for small $\nu$. This scaling is obtained from a balance of the viscous term $\nu \mathbf{q}^{2} \tilde{\mathbf{v}}$ in eq. (9) with the stretching term $\mathbf{v} \cdot \nabla \mathbf{U}$. A best fit results in

$$
q_{z}^{*} \simeq 0.8\left(\frac{U}{\nu \ell_{U}}\right)^{1 / 2}
$$

where we have kept $U=1$,and $\ell_{U}=1$ in the expression to recover the dimensions and the result is valid for small values of $\nu$ (large $\left.q_{z}^{*}\right)$. For layers of finite height $H$ the presence of these unstable modes depends on $H$. This is because the periodicity of the domain in the $z$ direction imposes that the wavenumber of a 3D perturbation satisfies $q_{z}=n / H$ where $n \geq 1$ is an integer. Therefore even for $\nu<\nu_{0}$ the flow will be stable to 3D perturbations if the layer thickness $H$ is thin enough so that $1 / H$ is larger than the largest unstable wavenumber $q_{z}^{*}$. 

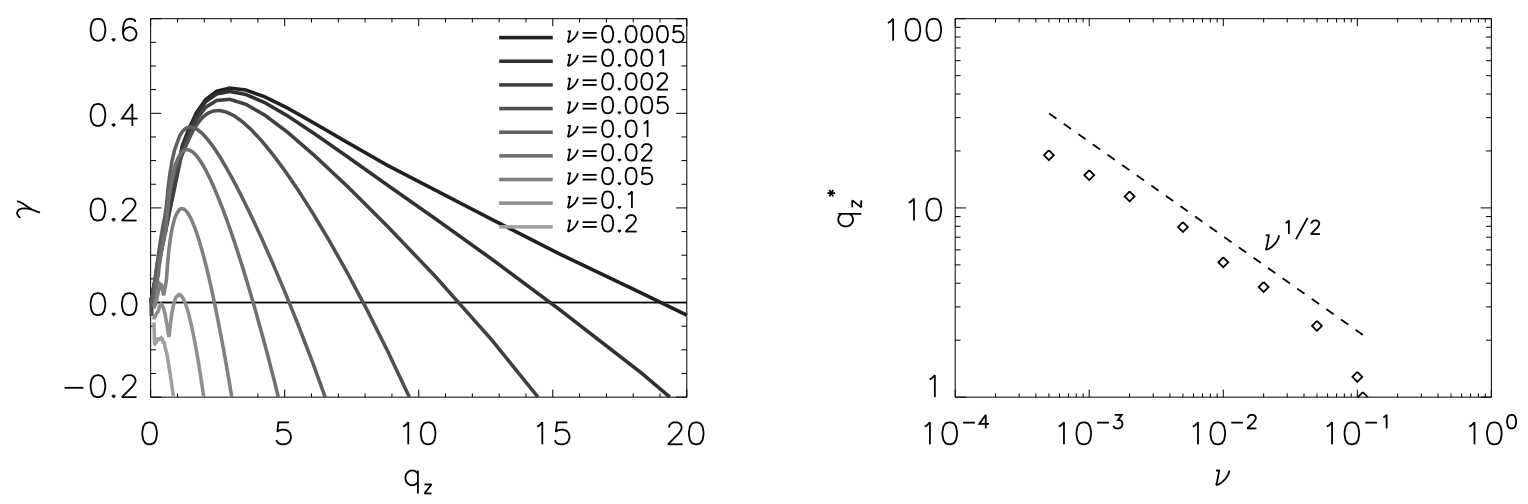

Figure 5. Left panel: The energy growth rate as a function of $q_{z}$. Right panel the critical wave number $q_{z}^{*}$ bellow which $q_{z}$ modes are unstable as a function of the viscosity.

\section{Two and three dimensional perturbations}

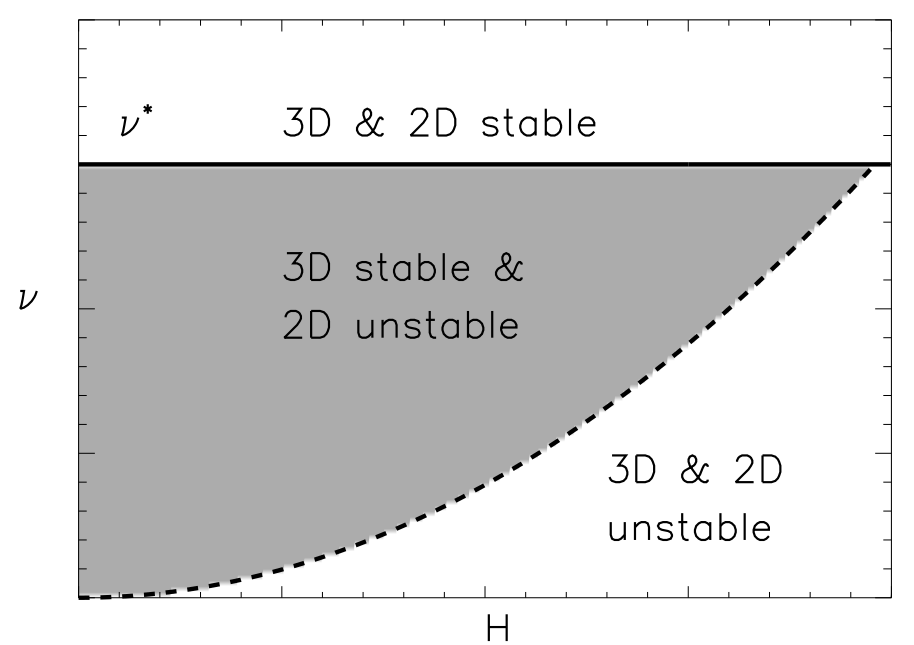

Figure 6. Stability diagram for a 2D flow

It is already possible to draw a first conclusion for 2D flows. From the analysis in section III A it was shown that the large $2 \mathrm{D}$ modes are unstable if the viscosity is small enough

$$
\nu<\nu_{*} \simeq 0.7 U \ell_{U}
$$

so that the eddy viscosity for 2D perturbations satisfies $\nu_{e d d y}+\nu<0$ (see eq. (17)). At the same time in section III B it is shown that $3 \mathrm{D}$ modes are stable if the layer is thin enough so that $1 / H$ is larger than maximum unstable wave number $q_{z}^{*}$ (see eq. (19)). This implies stability of the 3D modes if

$$
H \leq \frac{1}{q_{z}^{*}} \simeq \frac{\nu^{1 / 2}}{0.8\left(U k_{U}\right)^{1 / 2}} .
$$

Therefore there is a region is the parameter space $(H, \nu)$ determined by the conditions 20,21 where 3D modes are stable while $2 \mathrm{D}$ modes are unstable to large scale perturbations by an eddy viscosity mechanism. This is summarized in the diagram in figure 6 where condition 20 is given by the solid line while the condition 21 is marked by a dashed line. The region of 3D stable and 2D unstable modes is marked by gray. In this region a transfer of energy to the 
large scales is expected and possibly an inverse cascade can develop. Of course whether an inverse cascade builds up or not depends on the nonlinear evolution of the unstable modes and can not be determined by the stability of the laminar flow alone. Furthermore in the region that both 3D and 2D instabilities are present we can not certify nor exclude a transfer of energy to the large scales. This is because we can not a priori conclude if the nonlinear evolution of the unstable 3D modes will suppress or not the inverse transfer by the negative eddy viscosity mechanism.

\section{THREE DIMENSIONAL FLOWS}

This next section considers the stability of 3D laminar flows given in eq. (5) with in general $r \neq 0$. For these flows the minimum layer thickness $H$ one can consider is given by the periodicity of $\mathbf{U}$ in the $z$ direction $H=\ell_{U}$. Since the interest in this work is on thin layer for the remaining of this section we will fix $H$ to this minimum value. This limits the values of $q_{z}$ in the Floquet expansion to zero $q_{z}=0$ since all perturbations can only have vertical scales smaller than $H$. The investigation therefore will be restricted to large scale $2 \mathrm{D}$ perturbations with $\mathbf{q}$ of the form $\mathbf{q}=\left[q_{x}, 0,0\right]$. It is noted that setting $q_{z}=0$ does not imply that $\mathbf{v}=e^{i \mathbf{q} \mathbf{x}} \tilde{\mathbf{v}}$ is independent of the coordinate $z$ because the field $\tilde{\mathbf{v}}$ depends in principle in all coordinates $x, y, z$ having the same periodicity as $\mathbf{U}$. Therefore $3 \mathrm{D}$ perturbations can still be unstable and as it is shown later they play an important role.
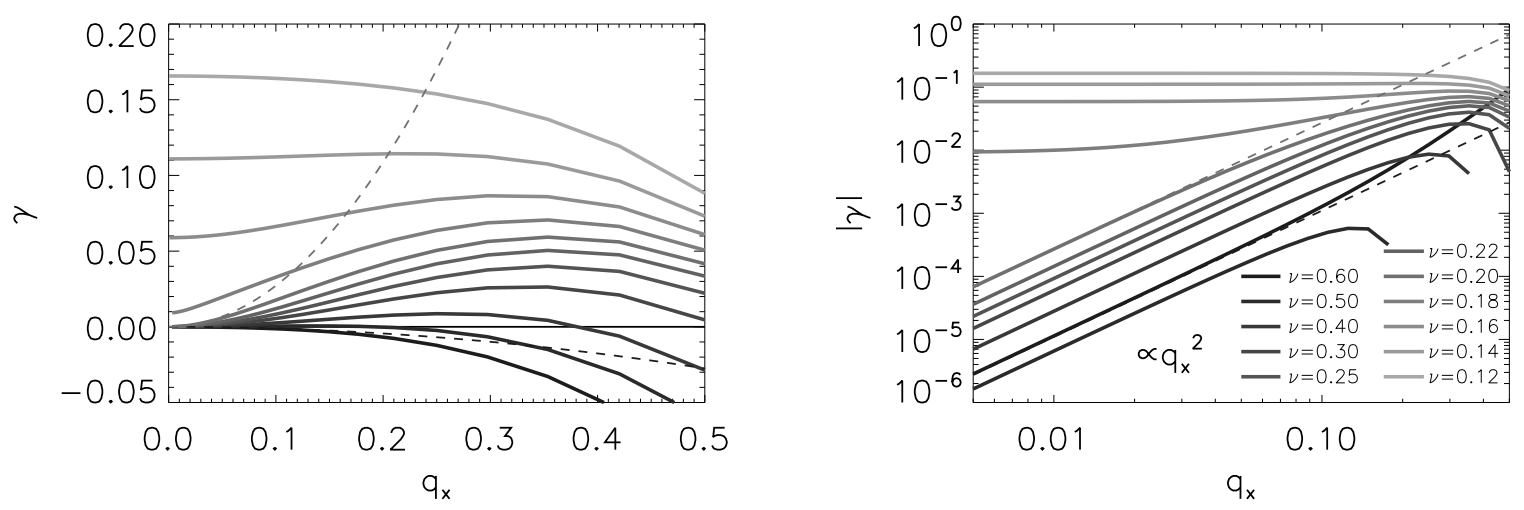

Figure 7. The growth rate $\gamma$ as a function of the wavenumber $q_{x}$ in linear scale (left panel) and logarithmic scale (right panel) for different values of $\nu$ and for $r=0.4$.

Figure 7 shows the measured growth rate as a function of the wavenumber $q_{x}$ for a flow with $r=0.4$ for different values of $\nu$. For large values of $\nu$ the growth rate shows a similar behavior as for the $r=0$ case shown in figure 1. For $\nu$ below a critical value the growth rate is negative and with a quadratic dependence in $q_{x}$ as in eq. (15). Above this critical value the growth rate retains its quadratic behavior (eq.15) but with a positive value of the proportionality coefficient $a$. This behavior can then again be interpreted as an effect of negative eddy viscosity such that $\gamma=-\left(\nu+\nu_{e d d y}\right) q_{x}^{2}$. However, if viscosity is further decreased the quadratic dependence on $q_{x}$ is lost and the growth rate attains a finite value at $q_{x}=0$.

A finite growth rate at $q=0$ can be a bit surprising since the growth of a non zero mean $\langle\mathbf{v}\rangle=\langle\tilde{\mathbf{v}}\rangle \neq 0$ field would violate momentum conservation. The contradiction is resolved by examining the relevant projection of the unstable mode to the large scales by looking at the ratio of $E_{0} / E=|\langle\tilde{\mathbf{v}}\rangle|^{2} /\left\langle|\tilde{\mathbf{v}}|^{2}\right\rangle$ that is shown in figure 8. This figure demonstrates that while for modes for which $\gamma \rightarrow 0$ as $q \rightarrow 0$ the ratio $E_{0} / E$ remains finite, for modes with $\gamma \neq 0$ as $q \rightarrow 0$ the projection to the large scales decreases as $E_{0} / E \propto q^{4}$. At $q=0$ therefore these last modes have a zero projection to the large scale modes, and correspond to purely small scales instabilities [32, 39].

Therefore the linear evolution of the large scale modes can be expressed in terms of an negative eddy viscosity up to this second critical value of $\nu=\nu_{3 D}$ bellow which 3D small scale instabilities begin. Large scale instabilities therefore are limited to values of the viscosity $\nu$ in the range $\nu_{*}>\nu>\nu_{3 D}$, where $\nu_{*}$ is the value above which large scales are stable, and for $\nu$ smaller than $\nu_{3 D}$ small scale instabilities are present. In principle for values of $\nu<\nu_{3 D}$ large scale modes $(0<q \ll 1)$ with positive growth rates $\gamma=-a\left(\nu+\nu_{\text {eddy }}\right) q^{2}>0$ still exist but their growth rate can be smaller than the growth rate of small scale instabilities of the $q=0$ modes. As a result for $\nu<\nu_{3 D}$ we can not predict if the flow would be able to transfer energy to the large scales with the linear evolution model given in 7 and a nonlinear theory for the upscale transfer of energy is required. 

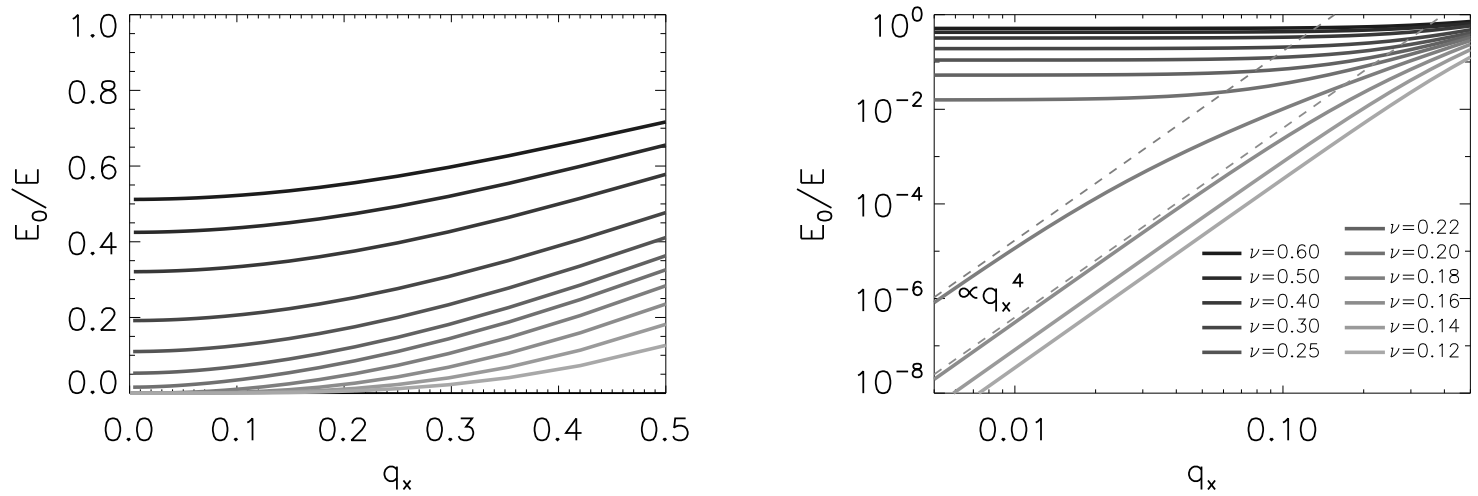

Figure 8. The ratio $E_{0} / E$ of the large energy $E_{0}=\frac{1}{2}|\langle\tilde{\mathbf{v}}\rangle|^{2}$ to the total energy of the perturbation $E=\frac{1}{2}\left\langle|\tilde{\mathbf{v}}|^{2}\right\rangle$ as a function of $q$, for different values of $\nu$ in linear scale (left panel) and logarithmic scale (right panel). The color-shade of the lines is the same for both panels.

In the range $\nu>\nu_{3 D}$, the value of the eddy viscosity can be evaluated by fitting to a parabola as was done in section III A. The values of the eddy viscosity for different values of $\nu$ and different values of $r$ were calculated and are plotted in the left panel of figure 9. This allows us to estimate the value of $\nu_{*}$ at which large scale $2 \mathrm{D}$ modes
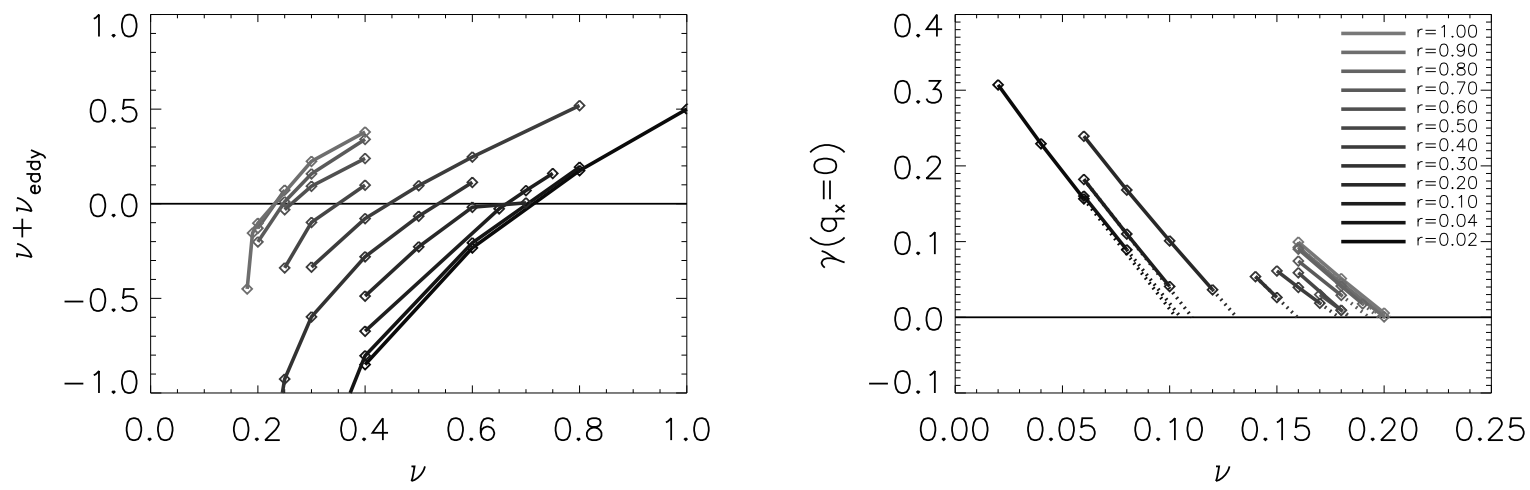

Figure 9. Left panel: Effective viscosity as a function of $\nu$ for the different values of $r$ Right panel: Small scale instability growth rate $\gamma(q=0)$ as a function of $\nu$ for different values of $r$.

become unstable. Similarly we can calculate the value $\nu_{3 D}$ by looking at the growth rate of small scale instabilities $q=0$ and determining by extrapolation at which value of $\nu$ the growth rate $\gamma(q=0)$ of small scale modes becomes positive. This is shown in the right panel of figure 9 for different values of $r$.

The end result is shown in figure 10 where the stability diagram of the parameter space is shown in the parameter space $(\nu, r)$. The space is split in three regions. One (marked by white) for which the growth rate of all instabilities is negative, one (marked by light gray) for which large scale modes are unstable by a negative eddy viscosity instability but 3D instabilities have negative growthrate, and finally one region (marked by dark gray) for which both small-scale and large-scale instabilities are present. For 2D flows $r=0$ the region for which the effective viscosity is negative, is larger. As $r$ is increased, the value of $\nu_{3 D}$ (dashed line) is increased and the value of $\nu_{*}$ (solid line) is decreased and as a result the range of values for which large scale instabilities can be observed is shrunk. Nonetheless even for $r=1$ for which the laminar flow does not contain any 2D part there is still a small range of values of $\nu$ for which only the large scale modes are unstable. This implies that transfer of energy to large scales can be observed in 3D flows as well. 


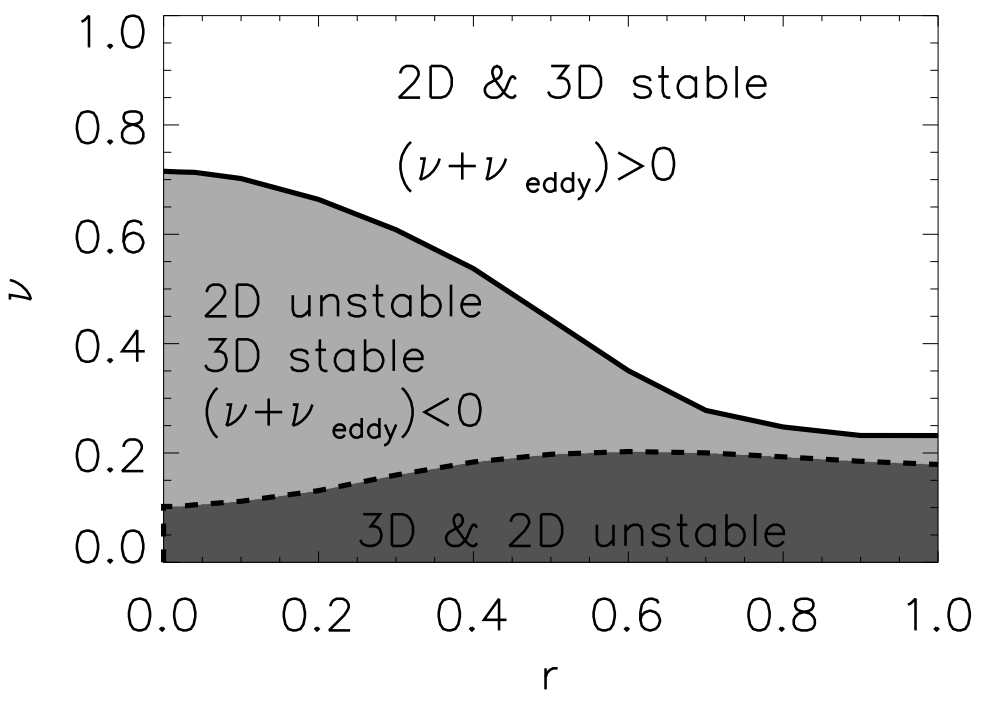

Figure 10. Stability diagram of the parameter space.

\section{CONCLUSIONS}

This work examined the linear stability of some basic linear flows against 2D large scale instabilities 3D small scale instabilities. The results showed that for the examined flows, 2D instabilities appeared first as the viscosity was decreased transferring energy to larger scales. The 3D instabilities appeared afterwards at smaller values of the viscosity and transfer energy to smaller scales. At sufficiently small viscosity therefore both types of instabilities are present and thus transfer to both large and small scales is possible.

In more detail, 2D laminar flows in thin layers become unstable to the large scale 2D modes due to an negative eddy viscosity type instability above a critical $R e$ independent of the layer height $H$. The onset of this instability is determined solely by the value of the eddy viscosity. The onset Reynolds number for 3D instabilities on the other hand increased as the layer thickness decreased. This result implies that for sufficiently thin layers the 2D part of the flow can be unstable and transfer energy to the large scales while 3D instabilities are suppressed. This result already indicates that depending on the layer thickness the flow can have different behaviors. For small $H$ it can behave like a 2D flow cascading energy inversely while if $H$ is large the flow can be dominated by 3D instabilities cascading energy forward. The stability diagram for the examined 2D flows is given in figure 6 .

For 3D flows it was shown that the negative eddy viscosity type of instabilities persist. Therefore, even for 3D flows there are unstable modes that move energy to larger scales. The large scale instabilities compete with the 3D small scale instabilities if $R e$ is above their onset. For the examined flows is was shown that $2 \mathrm{D}$ instabilities have in general a smaller onset $R e$ than the 3D instabilities, and thus a transfer to the large scales is still possible. However, as the flow becomes more 3D the difference between the two critical values decreases leaving little room to observe a large scale instability. These results are summarized in figure 10.

Finally we need to give a word of caution. The present results deal only with the stability of the laminar flows. Therefore we can only draw conclusions for the early stages of evolution of these instabilities. First, if the flow is 3D unstable and 3D turbulence develops then the transfer of energy to the large scales is not necessarily through an instability. A new nonlinear definition of an eddy-viscosity would be required if the notion of eddy-viscosity is at all valid in this case. Second, if the laminar flow is only $2 \mathrm{D}$ unstable then at the non-linear stage the flow can start to develop an inverse cascade $[1,9,10]$ and form condensates [42]. The stability properties these new states however will change and can be unstable to 3D perturbations. Interestingly, the shape of the condensate formed in a $2 \mathrm{D}$ inverse cascade in a square box is similar to the $2 \mathrm{D}$ flows examined here $[43,44]$ and the stability against 3D perturbations can be applied but they need to be examined for vortexes the size of the domain. In general however the co-existence of an inverse and forward cascade can not be neither confirmed or excluded just from the linear stability analysis. This would require a study of the nonlinear state of the system that can be studied in some cases with bounds [45, 46] or with the use of numerical simulations, and where the notion of eddy viscosity is not well established. 


\section{ACKNOWLEDGMENTS}

This work was granted access to the HPC resources of MesoPSL financed by the Region Ile de France and the project Equip@Meso (reference ANR-10-EQPX-29-01) of the programme Investissements d'Avenir supervised by the Agence Nationale pour la Recherche and the HPC resources of GENCI-TGCC-CURIE \& GENCI-CINES-JADE (Project No. A0010506421 \& A0030506421) where the present numerical simulations have been performed. This work has also been supported by the Agence nationale de la recherche (ANR DYSTURB project No. ANR-17-CE30-0004). The author would also like to thank A. van Kan and S. Benavides for their critical reading of this manuscript.

[1] A. Celani, S. Musacchio, and D. Vincenzi, "Turbulence in More than Two and Less than Three Dimensions," Physical Review Letters 104, 184506 (2010).

[2] R. Marino, P. D. Mininni, D. Rosenberg, and A. Pouquet, "Inverse cascades in rotating stratified turbulence: Fast growth of large scales," EPL (Europhysics Letters) 102, 44006 (2013).

[3] A. Pouquet and R. Marino, "Geophysical Turbulence and the Duality of the Energy Flow Across Scales," Physical Review Letters 111, 234501 (2013).

[4] Kannabiran Seshasayanan, Santiago Jose Benavides, and Alexandros Alexakis, "On the edge of an inverse cascade," Phys. Rev. E 90, 051003 (2014).

[5] E. Deusebio, G. Boffetta, E. Lindborg, and S. Musacchio, "Dimensional transition in rotating turbulence," Phys. Rev. E 90, 023005 (2014).

[6] Kannabiran Seshasayanan and Alexandros Alexakis, "Critical behavior in the inverse to forward energy transition in two-dimensional magnetohydrodynamic flow," Phys. Rev. E 93, 013104 (2016).

[7] A. Sozza, G. Boffetta, P. Muratore-Ginanneschi, and S. Musacchio, "Dimensional transition of energy cascades in stably stratified forced thin fluid layers," Physics of Fluids 27, 035112 (2015).

[8] R. Marino, A. Pouquet, and D. Rosenberg, "Resolving the Paradox of Oceanic Large-Scale Balance and Small-Scale Mixing," Physical Review Letters 114, 114504 (2015).

[9] Santiago Jose Benavides and Alexandros Alexakis, "Critical transitions in thin layer turbulence," Journal of Fluid Mechanics 822, 364-385 (2017).

[10] Stefano Musacchio and Guido Boffetta, "Split energy cascade in turbulent thin fluid layers," Physics of Fluids 29, 111106 (2017).

[11] D. Byrne, H. Xia, and M. Shats, "Robust inverse energy cascade and turbulence structure in three-dimensional layers of fluid," Physics of Fluids 23, $095109-095109$ (2011).

[12] Kannabiran Seshasayanan and Alexandros Alexakis, "Condensates in rotating turbulent flows," Journal of Fluid Mechanics 841, 434-462 (2018).

[13] A. Alexakis, "Rotating taylor-green flow," Journal of Fluid Mechanics 769, 4678 (2015).

[14] Antonio M Rubio, Keith Julien, Edgar Knobloch, and Jeffrey B Weiss, "Upscale energy transfer in three-dimensional rapidly rotating turbulent convection," Physical review letters 112, 144501 (2014).

[15] M. Shats, D. Byrne, and H. Xia, "Turbulence Decay Rate as a Measure of Flow Dimensionality," Phys. Rev. Lett. 105, $264501(2010)$.

[16] H. Xia, D. Byrne, G. Falkovich, and M. Shats, "Upscale energy transfer in thick turbulent fluid layers," Nature Physics 7, 321-324 (2011).

[17] Nicolas Francois, Hua Xia, Horst Punzmann, and Michael Shats, "Inverse energy cascade and emergence of large coherent vortices in turbulence driven by faraday waves," Physical review letters 110, 194501 (2013).

[18] Anna Frishman and Corentin Herbert, "Turbulence statistics in a 2d vortex condensate," arXiv preprint arXiv:1711.05536 (2017).

[19] A Alexakis and L Biferale, "Cascades and transitions in turbulent flows," Physics Reports (2018), https://doi.org/10.1016/j.physrep.2018.08.001.

[20] D. Byrne and J. A. Zhang, "Height-dependent transition from 3-D to 2-D turbulence in the hurricane boundary layer," Geophys. Rev. Lett. 40, 1439-1442 (2013).

[21] G.I. Taylor, "Eddy motion in the atmosphere," Phil. Trans. R. Soc. Lond. A 215, 1-26 (1915).

[22] Robert H Kraichnan, "Eddy viscosity in two and three dimensions," Journal of the atmospheric sciences 33, 1521-1536 (1976).

[23] Dieter Forster, David R Nelson, and Michael J Stephen, "Large-distance and long-time properties of a randomly stirred fluid," Physical Review A 16, 732 (1977).

[24] J-D Fournier and U Frisch, "Remarks on the renormalization group in statistical fluid dynamics," Physical Review A 28, 1000 (1983).

[25] Victor Yakhot and Steven A Orszag, "Renormalization group analysis of turbulence. i. basic theory," Journal of scientific computing 1, 3-51 (1986).

[26] Ye Zhou, George Vahala, and Murshed Hossain, "Renormalization-group theory for the eddy viscosity in subgrid modeling," Physical Review A 37, 2590 (1988). 
[27] A Yakhot, Steven A Orszag, V Yakhot, and M Israeli, "Renormalization group formulation of large-eddy simulations," Journal of Scientific computing 4, 139-158 (1989).

[28] Ye Zhou, George Vahala, and Murshed Hossain, "Renormalized eddy viscosity and kolmogorovs constant in forced navierstokes turbulence," Physical Review A 40, 5865 (1989).

[29] Massimo Germano, Ugo Piomelli, Parviz Moin, and William H Cabot, "A dynamic subgrid-scale eddy viscosity model," Physics of Fluids A: Fluid Dynamics 3, 1760-1765 (1991).

[30] Bérengère Dubrulle and Uriel Frisch, "Eddy viscosity of parity-invariant flow," Physical Review A 43, 5355 (1991).

[31] S Gama, M Vergassola, and U Frisch, "Negative eddy viscosity in isotropically forced two-dimensional flow: linear and nonlinear dynamics," Journal of fluid mechanics 260, 95-126 (1994).

[32] Alexandre Cameron, Alexandros Alexakis, and Marc-Étienne Brachet, "Large-scale instabilities of helical flows," Physical Review Fluids 1, 063601 (2016).

[33] U Frisch, Zh S She, and PL Sulem, "Large-scale flow driven by the anisotropic kinetic alpha effect," Physica D: Nonlinear Phenomena 28, 382-392 (1987).

[34] LD Meshalkin and Ia G Sinai, "Investigation of the stability of a stationary solution of a system of equations for the plane movement of an incompressible viscous liquid," Journal of Applied Mathematics and Mechanics 25, 1700-1705 (1961).

[35] AA Nepomnyashchy, "On the stability of the secondary flow of a viscous fluid in an infinite domain," Appl. Math. Mech 40, 886 (1976)

[36] G Sivashinsky and V Yakhot, "Negative viscosity effect in large-scale flows," The Physics of fluids 28, 1040-1042 (1985).

[37] Gregory I Sivashinsky and Alexander L Frenkel, "On negative eddy viscosity under conditions of isotropy," Physics of Fluids A: Fluid Dynamics 4, 1608-1610 (1992).

[38] G. I. Taylor and A. E. Green, "Mechanism of the production of small eddies from large ones," Proc. R. Soc. Lond. A 158, 499-521 (1937).

[39] Alexandre Cameron and Alexandros Alexakis, "Fate of alpha dynamos at large r m," Physical review letters 117, 205101 (2016).

[40] André Thess, "Instabilities in two-dimensional spatially periodic flows. part ii: Square eddy lattice," Physics of Fluids A: Fluid Dynamics 4, 1396-1407 (1992).

[41] Carlo Marchioro, "An example of absence of turbulence for any reynolds number," Communications in mathematical Physics 105, 99-106 (1986).

[42] Adrian van Kan and Alexandros Alexakis, "Condensates in thin-layer turbulence," arXiv preprint arXiv:1808.00578 (2018).

[43] Basile Gallet and William R Young, "A two-dimensional vortex condensate at high reynolds number," Journal of Fluid Mechanics 715, 359-388 (2013).

[44] Anna Frishman, Jason Laurie, and Gregory Falkovich, "Jets or vortices - what flows are generated by an inverse turbulent cascade?" Physical Review Fluids 2, 032602 (2017).

[45] B. Gallet and C. R. Doering, "Exact two-dimensionalization of low-magnetic-Reynolds-number flows subject to a strong magnetic field," Journal of Fluid Mechanics 773, 154-177 (2015).

[46] Basile Gallet, "Exact two-dimensionalization of rapidly rotating large-reynolds-number flows," Journal of Fluid Mechanics 783, 412-447 (2015). 